\title{
TBP2 is a general transcription factor specialized for female germ cells
}

\author{
Ferenc Müller* and Làszlò Tora†
}

\begin{abstract}
Addresses: *Department of Medical and Molecular Genetics, School of Clinical and Experimental Medicine, College of Medical and Dental Sciences, University of Birmingham, Birmingham B15 2TT, UK. †Department of Functional Genomics, Institut de Génétique et de Biologie Moléculaire et Cellulaire (IGBMC), UMR 7104 CNRS, UdS, INSERM U964, BP 10142, F-67404 Illkirch Cedex, France.
\end{abstract}

Correspondence: Ferenc Müller. Email: f.mueller@bham.ac.uk. Làszlò Tora. Email: laszlo@igbmc.u-strasbg.fr

\begin{abstract}
The complexity of the core promoter transcription machinery has emerged as an additional level of transcription regulation that is used during vertebrate development. Recent studies, including one published in BMC Biology, provide mechanistic insights into how the TATA binding protein (TBP) and its vertebrate-specific paralog TBP2 (TRF3) switch function during the transition from the oocyte to the embryo.
\end{abstract}

See research article http://www.biomedcentral.com/1741-7007/7/45

Regulation of initiation of transcription by RNA polymerase II (Pol II) is central to any developmental process. A key regulatory step in eukaryotic transcription initiation is the assembly of basal transcription apparatus at the core promoter. This regulatory step has been brought into the spotlight by the discovery of multiple promoter binding factors that assemble into different basal transcription factor complexes. These complexes have to be matched in the future to the diversity of core promoter types and features [1]. This apparent diversity points towards a dynamic regulatory role for this machinery [1], which is very poorly understood.

The preinitiation complex includes the core promoter, Pol II and the general transcription factors TFIIA, B, D, E, F and $\mathrm{H}$. Originally, the core promoter recognition factor TFIID, which is composed of TATA-binding protein (TBP) and 14 TBP-associated factors (TAFs), was thought to be ubiquitous. Functional and genetic studies revealed that TBP is not exclusively required for all protein-coding gene transcription in vertebrates [2]. In line with genetic observations, biochemical analyses revealed the existence of alternative initiation complexes that have been suggested to replace TFIID in several in vivo and in vitro systems [1-3]. The diversity in the components of transcription initiation machinery prompts the questions of why this diversity is present in metazoans and how the various initiation complexes act in parallel in a cell or the multicellular organism.
TBP has a crucial role in preinitiation complex assembly: nucleating the binding of TFIID to promoters. However, it is a member of a protein family, and other members of the TBP family, such as TBP-like factor (TLF or TBPL1/TRF2/ TRP) and TBP2 (or TRF3/TBPL2), have been shown to substitute for TBP to mediate Pol II and Pol III transcription. TBP2 is a vertebrate-specific paralog of TBP, with much higher similarity to TBP than TLF (TBP2 is about 90\% similar to TBP in its core domain). Consistent with this similarity, TBP2 can bind the TATA box, to interact with the other general transcription factors TFIIA and TFIIB and mediate Pol II transcription initiation in vitro, just as TBP can [3,4]. These properties of TBP2 suggest a function complementary to that of TBP and raise the question of whether TBP and TBP2 are functional equivalents or carry out specialized functions. Thus, given the high level of similarity in biochemical properties between $\mathrm{TBP}$ and $\mathrm{TBP} 2$, the cause and mechanism for the retention of TBP2 following gene duplication remains to be explained. Two recent publications studying TBP2 function in frogs [5] and mice [6] provide some answers to this intriguing problem.

\section{Replacement of TBP by TBP2 in Xenopus oocyte transcription}

The transition from maternal to zygotic gene activation in the embryo has been a tractable and informative model system for studying the function of TBP family proteins in vertebrate ontogeny. Knockdown studies in Xenopus and zebrafish embryos showed that TBP and TBP2 are both indispensable for embryonic development and are both required for activation of zygotic genes [4,7]. Unexpectedly, TBP2 was shown to have a specialized role restricted to the ventral side of the embryo [4,7] and in hematopoiesis [8]. However, these results did not shed light on why TBP2 seemed to be mostly expressed in the female gonad in frogs and why only a low level of expression was detected in frog and fish embryos [4,5,7]. The apparent enrichment for TBP2 in the female gonad contrasted with opposing dynamics of TBP, suggesting a general feature for specific activity of TBP2 in the ovary in anamniotes [4,7]. 
To address the function of $\mathrm{TBP} 2$, in their recent $B M C$ Biology paper Akhtar and Veenstra [5] have investigated the role of $\mathrm{TBP} 2$ in oocyte transcription and compared it with that of TBP. They show that Xenopus oocytes lack TBP protein, whereas TBP2 is the major TBP-type factor in the germ cells. Later, in eggs and early embryos, TBP2 levels decrease, whereas TBP starts to accumulate after meiotic maturation and during cleavage stages of development (Figure 1). A major role for TBP2 in oocyte transcription was suggested by the observation that TBP2 is recruited to the transcriptionally active loops of the expanded 'lampbrush' chromosomes found in frog oocytes. The authors [5] exploited an overexpression system to show that in oocytes TBP2 is recruited to transcribed promoters together with Pol II. In addition, the authors [5] show that TBP2 is also recruited to Pol III promoters, further suggesting that $\mathrm{TBP} 2$ probably replaces $\mathrm{TBP}$ in mediating transcription by all three RNA polymerases.

The results described by Akhtar and Veenstra [5] highlight the functional significance of the apparent differentially available pool of TBP and TBP2 in oocytes and provide mechanistic insights into the dynamics of TBP and TBP2 protein. At the end of oocyte maturation TBP2 is degraded and transcription is globally repressed. By analyzing whether TBP2 degradation is directly linked to this change in general transcription, they show [5] that the repression is established by the germinal vesicle breakdown stage of oocyte development, a stage at which TBP2 degradation has already started but has not reached its maximum. Thus, it seems that it is not TBP2 degradation, but rather a loss of association of TBP2 with promoters, that coincides with transcriptional repression during meiotic maturation. This argues against a direct role for TBP2 degradation in the global shutdown of transcription during oocyte maturation. This conclusion, together with the observed reduction of TBP2 and enrichment of TBP in embryos, implies that the primary role of $\mathrm{TBP} 2$ degradation is to facilitate factor switching and transcriptional regulation during subsequent development (Figure 1).

The hypothesis of factor switching during the transition from maternal gene activity is consistent with a series of observations made in several vertebrate models ([2] and references therein). The picture emerging from the study by Akhtar and Veenstra [5] is that post-transcriptional regulation of $\mathrm{TBP}$ and $\mathrm{TBP} 2$ is important for regulating steady-state levels of TBP paralogs in frogs. This regulation results in striking differences in protein availability, and suggests a model for subfunctionalization (division of functions) of TBP paralogs between oocytes and embryos.

\section{A requirement for mouse TBP2 in female germ cell development}

The conclusions drawn from studies in frog oocytes [5] match those stemming from expression analysis and recent genetic loss-of-function studies carried out in mice $[6,9,10]$. In mice, the expression of Tbp2 mRNA has been detected specifically in the oocytes [4,9]. Although there is controversy regarding the specificity of this expression, a recent genetic study indicated that the main role of TBP2 is restricted to the female germline $([3,10]$ and references therein). $\mathrm{TBP} 2$ protein accumulates in the nuclei of growing mouse oocytes during folliculogenesis, and its level declines on ovulation to become undetectable after fertilization [10] (Figure 1). In contrast, TBP is expressed in the oocytes only at the beginning of folliculogenesis and after fertilization, but not during oocyte growth [10] (Figure 1).

Consistent with a specific expression in the ovary, $\mathrm{Tbp}^{-/}$ mice are viable and show no obvious phenotype [6]. However, females lacking TBP2 are sterile as a result of defective folliculogenesis. Tbp2 $\%$ females lack fully grown germinal-vesicle-stage oocytes and Pol II transcription is perturbed mainly at the primary follicle stage, when wildtype oocytes show extensive transcriptional activity. A general decrease in transcription is indicated by the reduced phosphorylation on serine 2 of Pol II and reduced methylation of histone $\mathrm{H}_{3}$ lysine 4, which are markers of active genes. Consequently, a significant number of oocytespecific genes are severely deregulated in $\mathrm{Tbp}^{-/-}$females. In agreement with the idea that $\mathrm{TBP} 2$ is the sole TBP-type factor in oocytes, TBP is dispensable for correct oocyte maturation and fertilization. In contrast, when TBP2 is misexpressed in early mouse embryos, where it is normally not expressed, it has a negative effect on cell proliferation, leading to developmental arrest [6]. These data together demonstrate that TBP2 is not required for mouse viability but has a critical and specialized role in mammalian female germ cell development, and they provide evidence for nonredundant functions of $\mathrm{TBP} 2$ and TBP in vivo in the mouse.

\section{TBP2 as a vertebrate oocyte-specific TBP- type factor}

The observations from frogs and mice [5,6] clearly establish TBP2 as an oocyte-specific TBP-type factor in vertebrates. In both organisms during certain stages of oocyte development, TBP is absent and dispensable (Figure 1). Thus, the unique role of TBP2 in oocyte transcription, in a highly specialized cell type, provides evidence that the basal transcription machinery is highly flexible and can switch factors depending on the cellular and ontogenic requirements.

A common model for subfunctionalization of TBP and TBP2 during the transition between oocyte and embryo is thus emerging from two evolutionarily distant vertebrates, although there remain important lineage-specific differences between them. In anamniotes, TBP2 proteins mostly (although not completely) degrade before the embryo is 


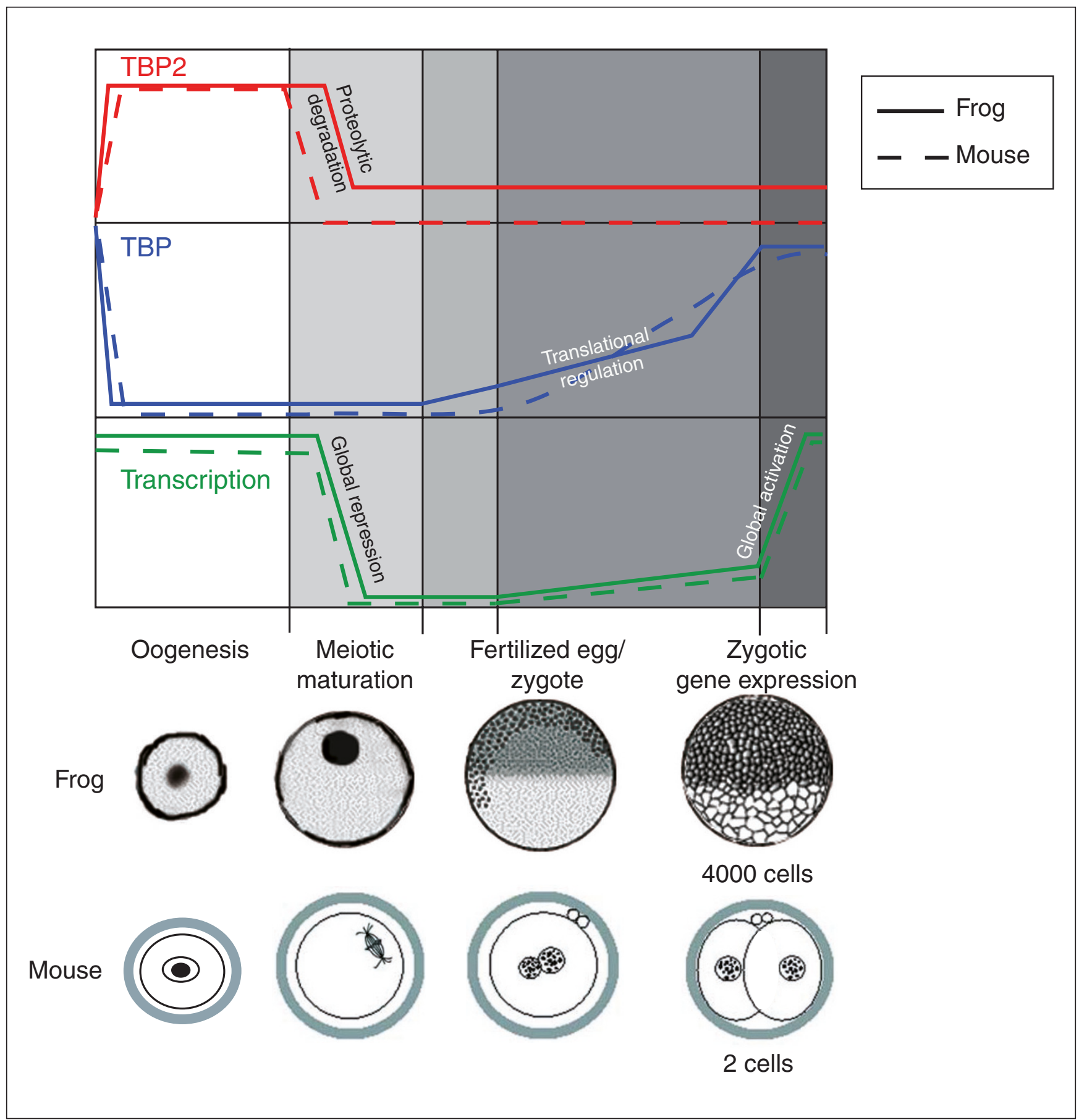

Figure 1

Regulation of TBP and TBP2 during oogenesis and the early stages of embryogenesis in vertebrates. Continuous line, frog; dashed line, mouse; red, TBP2; blue, TBP; green, general transcription. Stages are represented at the top by light to dark shading, and at the bottom by schematic representations. At most stages of oogenesis only TBP2 is expressed, which promotes oocyte-specific transcription during these stages. Upon meiotic maturation, TBP2 is actively degraded following global repression of transcription in maturing oocytes (as has been demonstrated in frogs). After fertilization and during the early stages of embryogenesis, TBP expression reaches the maximum levels that are needed to start zygotic transcription. In frogs zygotic transcription is largely delayed until the mid blastula and this process is regulated by late translation of maternal stores of tbp mRNA. In frog (and zebrafish) there are low levels of TBP2 during early stages of embryogenesis, whereas in mice no TBP2 has been detected during embryogenesis. Global zygotic transcription initiation is delayed in both frog and mouse, albeit to different developmental stages, and trace levels of zygotic transcription have been detected in both species before global genome activation. The figure has been generated by summarizing experiments described in $[4-7,9,10]$. 
formed [5]. In contrast to mammals, a large amount of TBP mRNA is produced maternally and seems to be prevented from being translated in the oocyte and the early embryo. To achieve factor switching, the maternal TBP mRNA translation is activated before global zygotic gene activation to generate an abundant pool of TBP protein, thereby becoming the dominant factor in the embryo.

The question remains: why is there a distinct requirement for either of the two TBP paralogs in oocytes and embryos? The high level of divergence of the amino termini between TBP2 and TBP may hold the key to this question. One possibility would be that the amino-terminal domain of $\mathrm{TBP} 2$ could determine the association of $\mathrm{TBP} 2$ with a special set of TAFs and/or other oocyte-specific factors that, in turn, would confer the oocyte-specific core promoter binding function to a non-canonical TFIID complex. Thus, a specialized TBP2-containing TFIID-like complex could act to mediate transcription from oocyte-specific genes and, in contrast to TBP, could inhibit cell cycle regulatory genes. Alternatively, the amino-terminal domain of TBP2 could function to regulate the DNA binding function of the carboxy-terminal domain, or to regulate protein dynamics, which as suggested by Akhtar and Veenstra [5] involves regulation of protein degradation.

In summary, a protein very similar to TBP seems to have evolved by gene duplication and has a non-redundant regulatory function in transcription initiation in the vertebrate oocyte. Further investigations are required to address how TBP2 functions in the oocyte and what specific properties and molecular mechanisms of transcription initiation distinguish the oocyte from the soma and the embryo.

\section{Acknowledgements}

We thank $S$ Bour for the illustration and ME Torres-Padilla for critically reading the manuscript. We apologize to colleagues whose work could not be cited owing to space and reference limitations and was only covered by reviews instead. This work was supported by a EUTRACC grant (LSHG-CT-2007-037445).

\section{References}

1. Juven-Gershon T, Kadonaga JT: Regulation of gene expression via the core promoter and the basal transcriptional machinery. Dev Biol 2009, [Epub ahead of print].

2. Jones KA: Transcription strategies in terminally differentiated cells: shaken to the core. Genes Dev 2007, 21:21132117.

3. Deato MD, Marr MT, Sottero T, Inouye C, Hu P, Tjian R: MyoD targets TAF3/TRF3 to activate myogenin transcription. Mol Cell 2008, 32:96-105.

4. Bartfai R, Balduf C, Hilton T, Rathmann Y, Hadzhiev Y, Tora L, Orban L, Muller F: TBP2, a vertebrate-specific member of the TBP family, is required in embryonic development of zebrafish. Curr Biol 2004, 14:593-598.

5. Akhtar W, Veenstra GJ: TBP2 is a substitute for TBP in Xenopus oocyte transcription. BMC Biol 2009, 7:45.

6. Gazdag E, Santenard A, Ziegler-Birling C, Altobelli G, Poch O, Tora L, Torres-Padilla ME: TBP2 is essential for germ cell development by regulating transcription and chromatin condensation in the oocyte. Genes Dev 2009, 23:2210-2223.

7. Jallow Z, Jacobi UG, Weeks DL, Dawid IB, Veenstra GJ: Specialized and redundant roles of TBP and a vertebratespecific TBP paralog in embryonic gene regulation in Xenopus. Proc Natl Acad Sci USA 2004, 101:13525-13530.

8. Hart DO, Raha T, Lawson ND, Green MR: Initiation of zebrafish haematopoiesis by the TATA-box-binding protein-related factor Trf3. Nature 2007, 450:1082-1085.

9. Xiao L, Kim M, DeJong J: Developmental and cell type-specific regulation of core promoter transcription factors in germ cells of frogs and mice. Gene Expr Patterns 2006, 6: 409-419.

10. Gazdag E, Rajkovic A, Torres-Padilla ME, Tora L: Analysis of TATA-binding protein 2 (TBP2) and TBP expression suggests different roles for the two proteins in regulation of gene expression during oogenesis and early mouse development. Reproduction 2007, 134:51-62.

Published: 30 November 2009

doi:10.1186/jbiol196

(C) 2009 BioMed Central Ltd 